\title{
Time dependence of the segmental relaxation time of poly(vinyl acetate)-silica nanocomposites
}

\author{
Virginie M. Boucher, ${ }^{1}$ Daniele Cangialosi, ${ }^{1,{ }^{*}}$ Angel Alegría, ${ }^{1,2}$ and Juan Colmenero ${ }^{1,2,3}$ \\ ${ }^{1}$ Centro de Física de Materiales (CSIC-UPVIEHU), Paseo Manuel de Lardizabal 5, 20018 San Sebastián, Spain \\ ${ }^{2}$ Departamento de Física de Materiales, Universidad del País Vasco (UPV/EHU), Apartado 1072, 20080 San Sebastián, Spain \\ ${ }^{3}$ Donostia International Physics Center, Paseo Manuel de Lardizabal 4, 20018 San Sebastian, Spain
}

(Received 26 July 2012; published 1 October 2012)

\begin{abstract}
The aging-time dependence of the segmental relaxation time of poly(vinyl acetate) (PVAc) in the glassy state is investigated in the bulk polymer and its nanocomposites with silica $\left(\mathrm{SiO}_{2}\right)$. These systems present identical segmental dynamics, when this is probed in the equilibrium supercooled liquid by broadband dielectric spectroscopy. An acceleration of the physical aging process of $\mathrm{PVAc}$ with $\mathrm{SiO}_{2}$ was detected by monitoring the enthalpy recovery through differential scanning calorimetry. The segmental relaxation time during physical aging, followed by means of BDS, has been shown to increase more rapidly the higher the $\mathrm{SiO}_{2}$ concentration in PVAc is. Thermally stimulated depolarization current experiments show that this is the case over the whole probed glassy state. This means that nanocomposites displaying a relatively slow segmental mobility evolve toward equilibrium more rapidly than the bulk. Furthermore, despite the faster increase in the relaxation time with aging time, so-called self-retardation, the nanocomposites and their bulk counterpart reach the same values of equilibrium relaxation time. These findings not only confirm the assumption of identical equilibrium dynamics even in the aging regime for all nanocomposites and bulk polymers, proposed in previous works, but also highlight the fact that the physical aging rate is not determined solely by the polymer segmental dynamics, the amount of interface being an additional relevant parameter.
\end{abstract}

DOI: 10.1103/PhysRevE.86.041501

PACS number(s): 64.70.pj, 36.20.-r, 81.05.Qk, 81.40.Tv

\section{INTRODUCTION}

The last 15 years have seen an increasing interest in polymer nanocomposites, materials resulting from the association of a polymer matrix with inorganic fillers with at least one dimension $\leqslant 100 \mathrm{~nm}[1,2]$. These materials are particularly attractive due to the combination, and sometimes the synergy, of the properties relative to both components [3]. The so-obtained unique properties of polymer nanocomposites make them interesting in many application fields and present several intriguing aspects from a fundamental point of view. Relative to the vast amount of literature dealing with the effect of inorganic fillers on the physical (mechanical, optical, electrical, etc.) properties of polymers, little has been reported on the long-term performances of polymer nanocomposites, in particular, those depending on the physical aging behavior of these materials [4], a temperature- and time-dependent process.

Physical aging is a ubiquitous phenomenon in amorphous glasses. These are generally found in an unstable thermodynamic state after their cooling from the equilibrium liquid state. In an attempt to approach thermodynamic equilibrium, amorphous polymers undergo a slow reorganization towards a more stable structure, thus reducing the entropy (or enthalpy, volume) of the system $[5,6]$. This leads to a densification of the polymer, which affects the physical properties of the materials (e.g., mechanical, dielectric, transport properties) [7-9] and may have dramatic consequences for applications. The recent explosion of industrial use of polymer nanocomposites has not only raised the question of the effect of inorganic nanofillers on the long-term performance of polymers $[10,11]$, but also

*swxcacad@ehu.es revealed the need for a fundamental understanding of the physical aging process in such materials.

In previous studies, the physical aging of glassy polymers was shown to be affected by the addition of inorganic nanofillers [12-15]. In particular, it was found that the polymer reaches a relative thermodynamic equilibrium after shorter aging times with increasing weight fraction of fillers [13-16]. In enthalpy recovery studies, the amount of recovered enthalpy was observed to decrease with the presence of fillers. However, enthalpy recovery studies allow the achievement of only relative values of enthalpy, which do not give any insight into the respective equilibrium reached by each sample. Such information should be extracted using techniques that provide absolute values of some material's property directly affected by the densification occurring during physical aging. Furthermore, in previous studies an acceleration of physical aging was found in glassy polymer nanocomposites whose segmental dynamics in the melt state was unchanged by the addition of fillers. This was concluded from broadband dielectric spectroscopy (BDS) results on the equilibrium segmental dynamics at temperatures somewhat higher than those employed for physical aging studies. Thus, these findings questioned the widespread idea that the physical aging rate-in terms of the time needed to reach relative equilibriumis solely related to the segmental dynamics $[5,6]$, at least for polymer nanocomposites. It was assumed that, in these systems, other parameters such as the amount of nanofillers in the glassy polymer, play a role in the determination of the physical aging rate, whose characteristic time can thus be written as $\tau_{\mathrm{PA}}=\tau_{\alpha} f_{A / V}$, where $\tau_{\alpha}$ is the segmental relaxation time, and $f_{A / V}$ is a function related to the area of filler-tovolume of polymer ratio, $A / V$, which means the amount of interface polymer-filler. However, no information on the fate of the segmental relaxation time during the course of aging was provided. On this basis, the idea of monitoring the time 
evolution of the segmental relaxation time in a bulk glassy polymer and its nanocomposites appeared as a means to check this relation between $\tau_{\mathrm{PA}}$ and $\tau_{\alpha}$ via $f_{A / V}$, on the one hand, and to provide direct evidence of the achievement of a similar equilibrium state below the conventional $T_{g}$ for the bulk and its nanocomposites, on the other.

To do so, poly(vinyl acetate) (PVAc)-silica $\left(\mathrm{SiO}_{2}\right)$ nanocomposites were prepared and characterized in terms of $\mathrm{SiO}_{2}$ weight fraction and $\mathrm{SiO}_{2}$ nanoparticle dispersion. The invariance of PVAc segmental dynamics in the presence of $\mathrm{SiO}_{2}$ nanoparticles was checked by BDS. The speeding-up of the physical aging of PVAc in nanocomposites was confirmed by monitoring the enthalpy recovery by means of differential scanning calorimetry (DSC). The aging-time dependence of the segmental relaxation time of PVAc in the bulk and in nanocomposites was monitored by the thermally stimulated depolarization current (TSDC) and BDS. The so-obtained results are compared and discussed in the light of a physical aging mechanism driven not only by segmental dynamics, but also by the amount of polymer-filler interfaces in the case of polymer nanocomposites.

\section{EXPERIMENTAL PROCEDURE}

\section{A. Sample preparation and particle dispersion characterization}

PVAc was purchased from Aldrich, Inc. The molecular weight was $M_{w}=194800 \mathrm{~g} \mathrm{~mol}^{-1}$, and the polydispersity $M_{w} / M_{n}=3.06$. Nanoparticles were Nan-O-Sil amorphous colloidal silica provided by Energy Strategy Associates, Inc. These are spherical particles with an average diameter of $100 \mathrm{~nm}$. Prior to mixing with the polymer, silica nanoparticles were functionalized in tetrahydrofuran (THF) with octadecyltrimethoxysilane (OTMS) to avoid particle aggregation. The polymer solution in THF and functionalized silica nanoparticles were subsequently solution blended in THF. The solution was then cast onto a clean glass substrate warmed up for faster solvent evaporation and left in a fume hood for at least $15 \mathrm{~h}$. The films were finally put in a vacuum oven at $353 \mathrm{~K}$ for at least $72 \mathrm{~h}$ in order to eliminate all traces of solvent. The silica content in the nanocomposite samples was measured by thermogravimetric analysis (TGA), on a thermogravimeter TA Q-500, with a heating ramp of $10 \mathrm{~K} \mathrm{~min}^{-1}$ up to $1023 \mathrm{~K}$ under flowing nitrogen $\left(20 \mathrm{~cm}^{3} \mathrm{~min}^{-1}\right)$. The values of silica weight fractions were found to be 8.1 and $24.6 \mathrm{wt} \%$. The corresponding volume fractions were, respectively, 4.2 and $12.9 \mathrm{vol} \%$. These samples are referred to as V8 and V25, respectively.

Transmission electron microscopy measurements were performed using a high-resolution transmission electron microscope, TECNAI G2 20 Twin. Measurements were carried out using an accelerating voltage of $200 \mathrm{kV}$, under low-dose conditions. Nanocomposite ultrathin sections of about $70 \mathrm{~nm}$ were cut by a LEICA EM UC6 microtome equipped with a diamond knife and placed on a 400-mesh copper grid. Figure 1 shows representative transmission electron micrographs for both $\mathrm{PVAc}_{-} \mathrm{SiO}_{2}$ nanocomposites. These images show a rather homogeneous dispersion of the nanoparticles into the PVAc matrix. In both samples, aggregation of silica particles is present. However, one can qualitatively observe the higher
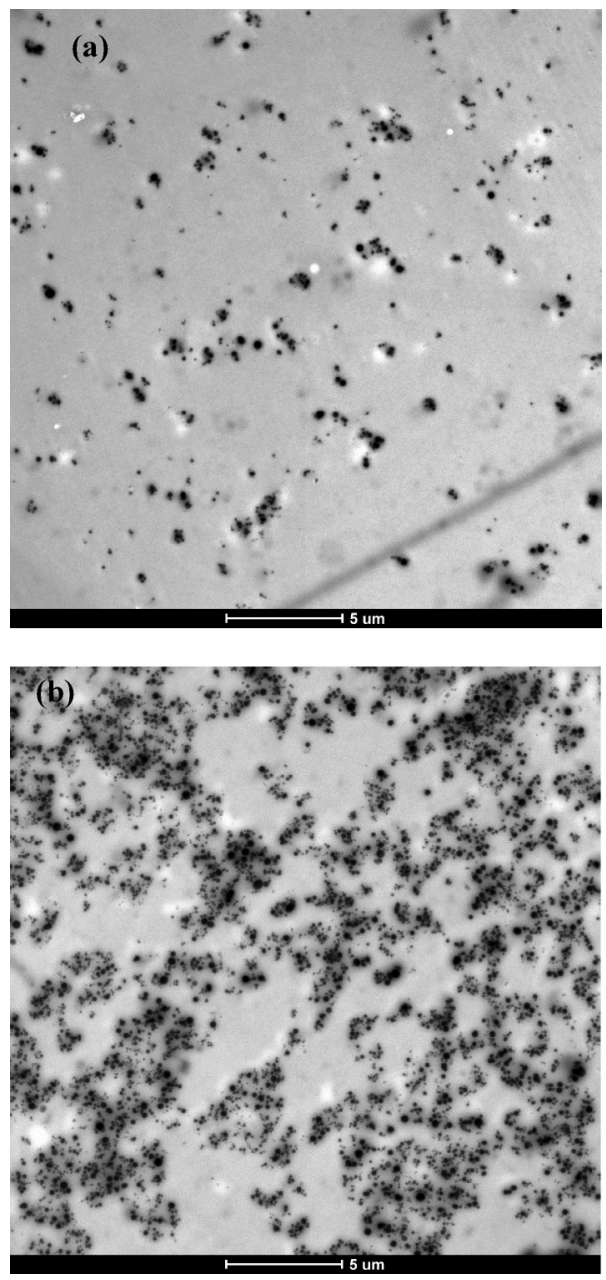

FIG. 1. Transmission electron microscopy images of $\mathrm{PVAc}-\mathrm{SiO}_{2}$ nanocomposite samples: (a) V8 and (b) V25.

amount of interface in the highly concentrated sample relative to the other one, which is the most important parameter concerning the particle dispersion in the framework of this study.

\section{B. Preliminary physical characterization: Segmental dynamics, enthalpy recovery, and $T_{g}$}

The molecular dynamics of the nanocomposites and native polymer was determined by means of BDS employing a a Novocontrol Alpha Analyzer. This allows measurement of the complex dielectric permittivity, $\epsilon^{*}=\epsilon^{\prime}(\omega)-i \epsilon^{\prime \prime}(\omega)$, where $\epsilon^{\prime}$ and $\epsilon^{\prime \prime}$ are, respectively, the storage and loss part of the dielectric permittivity, in the frequency range $10^{-3}$ $10^{7} \mathrm{~Hz}$. Samples with a thickness of about $200 \mu \mathrm{m}$ were placed between two gold-plated electrodes and introduced in the dielectric cell. The temperature was controlled by a Novocontrol Quatro cryosystem with a precision of $\pm 0.1 \mathrm{~K}$. All measurements were performed in a nitrogen atmosphere to avoid moisture uptake. Frequency scans were recorded on all samples isothermally in steps of $2.5 \mathrm{~K}$ from 313 to $377 \mathrm{~K}$. No effect of the previous thermal history was found on the mean relaxation time, evaluated as $\tau=1 / \omega_{\max }$, where $\omega_{\max }$ is the frequency corresponding to the maximum of the dielectric 
loss. Regarding the study of the dynamics in the glassy state (at $T<T_{g}=314.2 \mathrm{~K}$, from the midpoint of the DSC curve of bulk PVAc upon cooling at $20 \mathrm{~K} \mathrm{~min}^{-1}$ ), the frequency range was extended to $10^{-3} \mathrm{~Hz}$. Moreover, in order to probe their equilibrium dynamics, the samples were first annealed for $2 \mathrm{~h}$ at the studied temperature before data recording. This time proved to be sufficient to achieve thermodynamic equilibrium even at the lowest investigated temperature $(311.6 \mathrm{~K})$.

Thermal analysis was performed by means of DSC-Q2000 from TA Instruments calibrated with melting indium. DSC measurements were carried out on a mass of 5-10 $\mathrm{mg}$ for all samples in nitrogen atmosphere. For enthalpy recovery measurements, all experiments began with a heating ramp to a temperature of $T_{g}+40 \mathrm{~K}$ in order to erase the material's previous thermal history. The samples were subsequently cooled down at a programmed rate of $20 \mathrm{~K} \mathrm{~min}^{-1}$ to reach $T_{g}-60 \mathrm{~K}$ before being stabilized at the temperature used for annealing in the calorimeter (here $T_{a}=305.6,308.1$, or $309.6 \mathrm{~K}$ ), for times from several minutes to several days, before being cooled to $T_{g}-60 \mathrm{~K}$ at a cooling rate of $20 \mathrm{~K} \mathrm{~min}^{-1}$ and, finally, reheated at $10 \mathrm{~K} \mathrm{~min}^{-1}$ for data recording. Second scans were performed immediately after a new quench at $20 \mathrm{~K} \mathrm{~min}^{-1}$. The corresponding procedure has been schematized in a previous work [14]. The amount of enthalpy relaxed during aging (and thus recovered during the DSC scan) for a period of time $t_{a}$ at $T_{a}$ was evaluated by integration of the difference in thermograms of aged versus unaged samples, according to the equation

$$
\Delta H\left(T_{a}, t_{a}\right)=\int_{T_{x}}^{T_{y}}\left(C_{p}{ }^{a}(T)-C_{p}{ }^{u}(T)\right) d T,
$$

where $C_{p}{ }^{a}(T)$ and $C_{p}{ }^{u}(T)$ are, respectively, the specific heat of the aged and unaged samples, and $T_{x}$ and $T_{y}$ are, respectively, temperatures below and above the calorimetric $T_{g}$, chosen as the temperatures at which the unaged and the aged sample scans superimpose below and above $T_{g}$, respectively. Typically, these temperatures were 303 and $333 \mathrm{~K}$ for PVAc-based samples.

The temperature of the glass transition onset, $T_{g}^{\text {on }}$, defined as the temperature at which nonequilibrium effects start to appear [17], has been determined for two cooling rates: 0.2 and $20 \mathrm{~K} \mathrm{~min}^{-1}$. In all cases, the samples were first equilibrated at $353 \mathrm{~K}$. Then the following procedure was employed. (i) Samples were cooled down at $0.2 \mathrm{~K} \mathrm{~min}^{-1} / 20$ $\mathrm{K} \mathrm{min}^{-1}$ to a selected temperature, then cooled down to $273 \mathrm{~K}$ at a rate of $20 \mathrm{~K} \mathrm{~min}^{-1}$ before being heated up at the selected temperature, where they were annealed for $45 \mathrm{~min}$. Samples were subsequently cooled down at $20 \mathrm{~K} \mathrm{~min}^{-1}$ to $273 \mathrm{~K}$, and a heating scan at $20 \mathrm{~K} \mathrm{~min}^{-1}$ was recorded until $353 \mathrm{~K}$. (ii) Afterwards, samples were cooled down at $0.2 \mathrm{~K} \mathrm{~min}^{-1} / 20 \mathrm{~K}$ $\min ^{-1}$ to the temperature at which they were previously annealed and, once this temperature was reached, immediately cooled down at $20 \mathrm{~K} \mathrm{~min}^{-1}$ to $273 \mathrm{~K}$, and a heating scan at $20 \mathrm{~K} \mathrm{~min}^{-1}$ was recorded until $353 \mathrm{~K}$. The procedure followed for the two cooling rates is schematized in a previous work [17]. In both cases, stages $i$ and ii delivered two specific heatversus-temperature plots, for the aged and the unaged samples, respectively. If the two plots overlap, the selected temperature is above the $T_{g}^{\text {on }}$. Conversely, if the plot corresponding to the aged sample displays an excess specific heat overshoot in comparison to the one corresponding to the unaged sample, the selected aging temperature lies below the $T_{g}^{\text {on }}$.

\section{Monitoring of the segmental relaxation time during physical aging}

TSDC measurements were performed using $10 \mathrm{~K} \mathrm{~min}^{-1}$ heating and cooling rates and a voltage of $50 \mathrm{~V}$. The voltage was applied at a relatively high polarization temperature, $T_{p}=$ $323 \mathrm{~K}\left(>T_{g}=314.2 \mathrm{~K}\right)$, where the polymer dipoles are able to orient quickly. The samples were subsequently cooled down at $10 \mathrm{~K} \mathrm{~min}^{-1}$ to the aging temperature, $T_{a}$ (here $T_{a}=$ $305.6 \mathrm{~K}, 308.1 \mathrm{~K}, 309.6 \mathrm{~K}<T_{g}=314.2 \mathrm{~K}$ ), at which they were annealed for various aging times, $t_{a}$, ranging from $1 \mathrm{~min}$ to several days. After this annealing, the samples were cooled again to a temperature low enough to assume that dipoles are frozen: the depolarization temperature, $T_{d}$ (here $T_{d}=223 \mathrm{~K}$ ), at which the poling voltage is switched off. The current due to depolarization is subsequently recorded upon heating the samples at $10 \mathrm{~K} \mathrm{~min}^{-1}$. For all experiments, the polarization conditions remained identical, apart from the annealing time at $T_{a}, t_{a}$. The corresponding procedure is schematized in Fig. 2. The segmental relaxation time $\left(\tau_{\alpha}\right)$ at a given aging time was evaluated from the temperature dependence of the depolarization current $[I(T)]$. For this the isothermal charge decay is assumed to follow the Kohlrausch-Williams-Watts (KWW) function [18]. In this framework the temperaturedependent current during a scan at heating rate $q=d T / d t$ is given by [19]

$$
I(T)=\beta \frac{Q(T)}{\tau_{\alpha}(T)}\left[\ln \left(\frac{Q_{0}}{Q(T)}\right)\right]^{(1-1 / \beta)},
$$

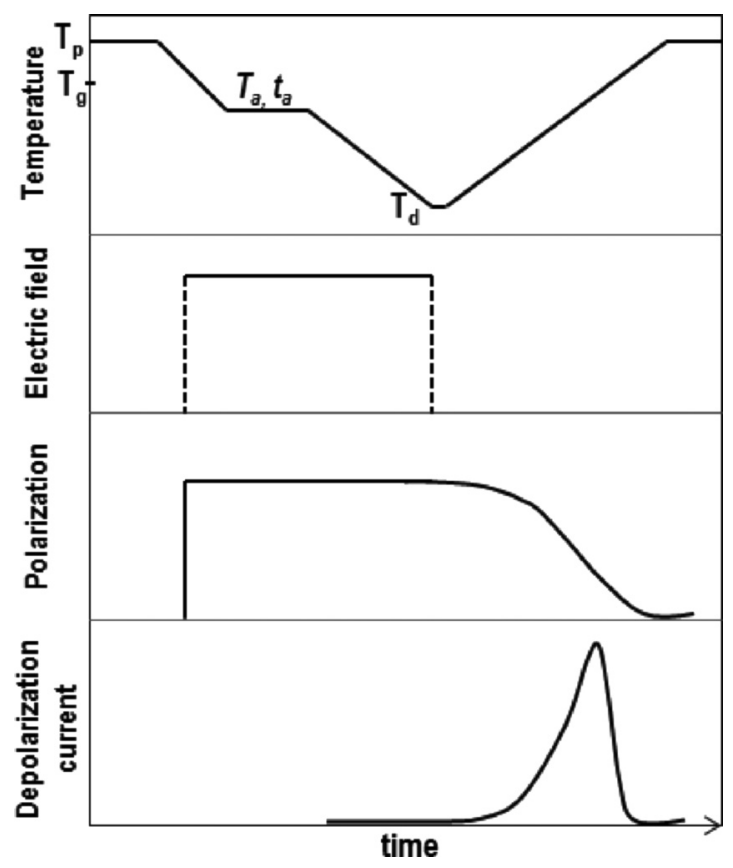

FIG. 2. Schematic of the time evolution of the different quantities involved in TSDC measurements carried out to follow the physical aging of bulk PVAc and its nanocomposites. 
where $Q(T)=(1 / q) \int_{T}^{T_{\max }} I\left(T^{\prime}\right) d T^{\prime}$ and $Q_{0}$ are the charge remaining at a given temperature and the total charge, respectively, $T_{\max }$ is the temperature corresponding to complete depolarization, and $\beta$ is the stretching exponent [18]. The latter, taken from Refs. [19] and [20], is $\beta=0.5$. Details on how Eq. (2) is obtained can be found elsewhere [19,21]. The relaxation time obtained from Eq. (2) is that corresponding to the KWW description. From this, $\tau_{\max }$, the most probable relaxation time, was determined following Refs. [22] and [23].

The monitoring of physical aging through BDS consisted in measuring continuously the time evolution of the dielectric spectrum of the samples at a fixed temperature: the aging temperature, $T_{a}\left(<T_{g}\right)$. To do so, the materials were first annealed for about $15 \mathrm{~min}$ at $373 \mathrm{~K}$ in order to erase their thermal history, then cooled down at $20 \mathrm{~K} \mathrm{~min}^{-1}$ to $173 \mathrm{~K}$, which ensured that the glass transition region was crossed at this imposed cooling rate, thus limiting the possible nonisothermal aging in this temperature region. The materials were subsequently heated up to the aging temperature at the rate of $20 \mathrm{~K} \mathrm{~min}^{-1}$. Once the sample temperature was stabilized at the aging temperature, the data were continuously recorded.

Despite the common use of the dielectric loss to monitor aging [12,24-33], it was chosen to monitor physical aging through the segmental relaxation time, which is the relevant quantity in this work. This parameter was extracted from the imaginary part of the dielectric spectra, by fitting them to the Havriliak-Negami (HN) function [34]:

$$
\epsilon^{\prime \prime}=\operatorname{Im}\left\{\frac{\Delta \epsilon}{\left[1+\left(i \omega \tau_{\mathrm{HN}}\right)^{\alpha_{\mathrm{HN}}}\right]^{\beta_{\mathrm{HN}}}}\right\} .
$$

Here $\Delta \epsilon$ is determined by extrapolating the values obtained at higher temperatures, in particular, those where the entire dielectric spectrum appears in the investigated frequency range. The $\alpha_{\mathrm{HN}}$ and $\beta_{\mathrm{HN}}$ parameters were fixed to fulfill the equation relating them to the stretching exponent, $\alpha_{\mathrm{HN}} \beta_{\mathrm{HN}}=$ $\beta^{1.23}$, and the equivalence between the $\mathrm{HN}$ and the KWW descriptions $[20,22,35]$. In doing so we obtain $\alpha_{\mathrm{HN}}=0.81$ and $\beta_{\mathrm{HN}}=0.51$. These parameters were then imposed for the fitting of the following dielectric spectra, so that only the structural relaxation time, $\tau_{\mathrm{HN}}$, was varying with time. Imposing a fixed value of $\Delta \epsilon$ at any aging time is based on the fact that its variation during physical aging is negligible in comparison to that of the segmental relaxation time. This point is considered in detail in Sec. III. From $\tau_{\mathrm{HN}}$, the most probable relaxation time $\tau_{\max }$ (that corresponding to the maximum of $\left.\epsilon^{\prime \prime}\right)$ was evaluated following Ref. [23].

As shown in the next section, the typical relaxation time at all investigated aging temperatures corresponds to frequencies $\left(<10^{-2} \mathrm{~Hz}\right)$ too low to be probed on a time scale shorter than that of physical aging. Thus only the high-frequency tail of the segmental relaxation was monitored and fitted during aging.

\section{RESULTS}

\section{A. Preliminary physical characterization}

Figure 3(a) displays the frequency dependence of the loss part of the dielectric permittivity for all investigated systems in equilibrium at $311.6 \mathrm{~K}$. Its temperature dependence at
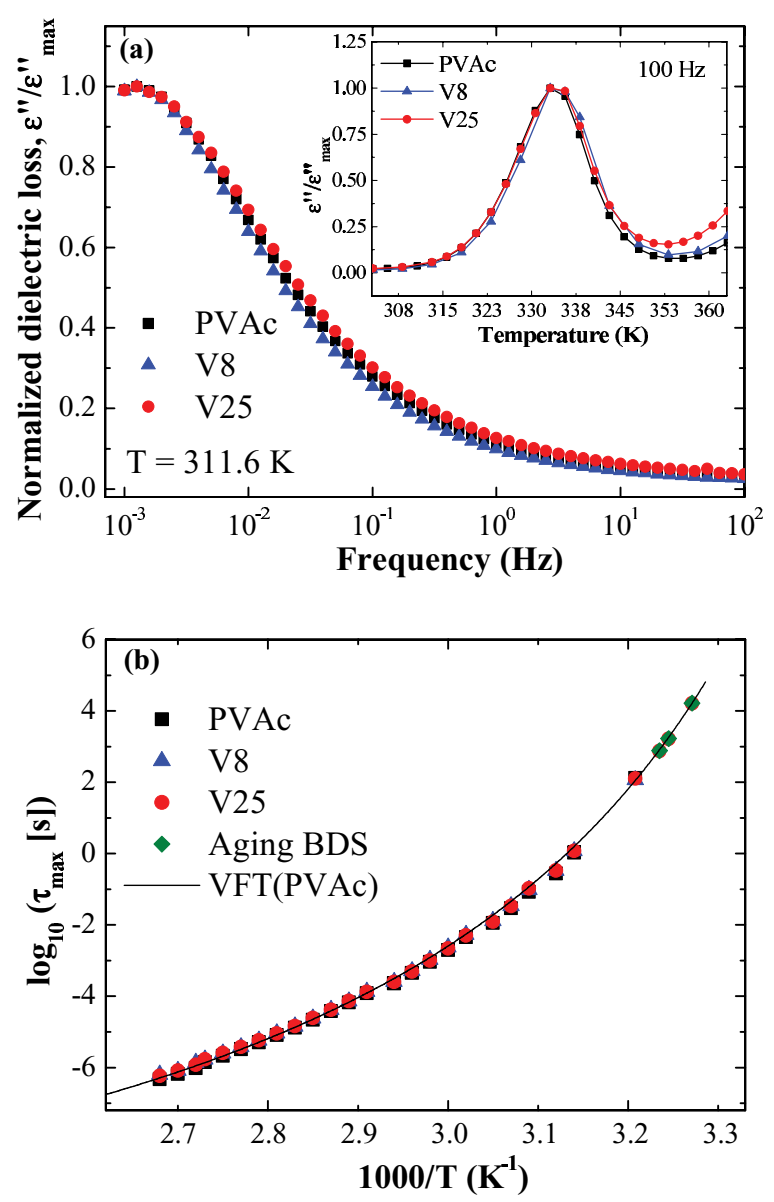

FIG. 3. (Color online) (a) Dielectric loss versus temperature for bulk PVAc and its nanocomposites with silica (V8 and V25) in equilibrium at a temperature of $311.6 \mathrm{~K}$. Inset: Dielectric loss versus temperature at the frequency of $100 \mathrm{~Hz}$. (b) Decimal logarithm of the relaxation time, $\tau_{\max }$, versus the reciprocal temperature of bulk PVAc and its nanocomposites with silica (V8 and V25). The line is the VFT fit to experimental data.

$100 \mathrm{~Hz}$ is presented in the inset. These figures indicate that PVAc segmental dynamics is unaffected by the presence of silica nanoparticles. This result concerns both the mean relaxation time, taken as the inverse of the frequency of $\epsilon^{\prime \prime}$ maximum, and the shape of the relaxation. Furthermore, Fig. 3(b), where the mean relaxation time is displayed as a function of the inverse of the temperature, indicates that the invariance of the segmental dynamics for all investigated systems holds throughout the temperature range investigated here. These results agree with those recently reported by Bogoslovov et al. [36]. Similar results have been found for other polymer nanocomposites with $\mathrm{SiO}_{2}[12,14,37]$. All segmental relaxation time data were fitted to the VogelFulcher-Tammann (VFT) equation, $\tau_{\max }(T)=\tau_{0} \exp (B /(T-$ $\left.T_{0}\right)$ ), with the fitting parameters $\tau_{0}=5 \times 10^{-13} \mathrm{~s}, B=1515$ $\mathrm{K}$, and $T_{0}=265 \mathrm{~K}$, in agreement with literature data $[20,38]$.

The physical aging of bulk PVAc and its nanocomposites with $\mathrm{SiO}_{2}$ was studied by following the enthalpy recovery of the materials after their annealing at a temperature $T_{a}\left(<T_{g}\right)$, for various periods of time $t_{a}$. The inset in Fig. 4 shows the temperature dependence of the specific heat after different 


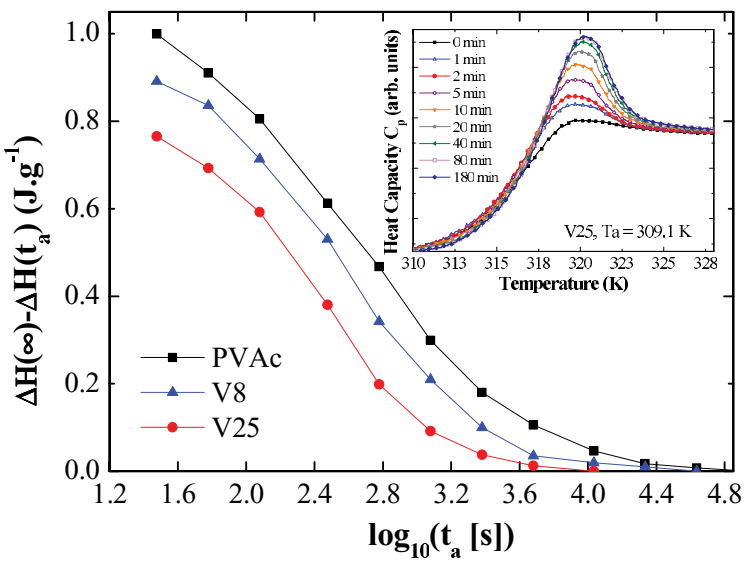

FIG. 4. (Color online) Distance from the recovered enthalpy $\Delta H\left(t_{a}\right)$ to the equilibrium value $\Delta H(\infty)$ as a function of aging time, at the aging temperature of $309.1 \mathrm{~K}$, for bulk PVAc and its nanocomposites. Inset: DSC heating scans of the sample V25 recorded after isothermal aging at $T_{a}=309.1 \mathrm{~K}$, for various aging times. Enthalpies are displayed on a per-gram-of-polymer basis.

annealing times at $309.1 \mathrm{~K}$ for the nanocomposite V25 as an example. These plots exhibit an endothermic overshoot in the glass transition range, which increases with the aging time. This represents the typical signature of physical aging. Figure 4 displays the time evolution of the distance of the recovered enthalpy to the value $\Delta H(\infty)$ [defined as the value of $\Delta H\left(t_{a}\right)$ at the plateau], obtained via Eq. (1) at $309.1 \mathrm{~K}$, for all investigated systems. While the line shape of the enthalpy recovery is generally independent of the filler fraction (all spectra can be superposed by horizontal shifting), the nanocomposites clearly exhibit faster enthalpy recovery. In particular, the amount of recovered enthalpy at a given aging time follows the order V25 $>\mathrm{V} 8>$ PVAc. This observation applies to all investigated temperatures as reported in Table I, where a comprehensive summary of the time to reach equilibrium, $t_{\infty}$, is presented. These results indicate a more efficient equilibration of nanocomposites in comparison to pure PVAc. Our results generally agree with a recent study on the enthalpy recovery of similar PVAc-silica nanocomposites [39].

The above observations on enthalpy recovery results are corroborated by $T_{g}^{\text {on }}$ data obtained as described in Sec. II and listed in Table I. PVAc- $\mathrm{SiO}_{2}$ nanocomposites exhibit a reduced $T_{g}^{\text {on }}$ in comparison to pure PVAc, and this reduction appears to be more pronounced for the nanocomposite V25. Furthermore, the $T_{g}^{\text {on }}$ reduction observed in the nanocomposites, though tiny, appears to be highly reproducible. Considering that $T_{g}^{\text {on }}$, as it is defined, marks the

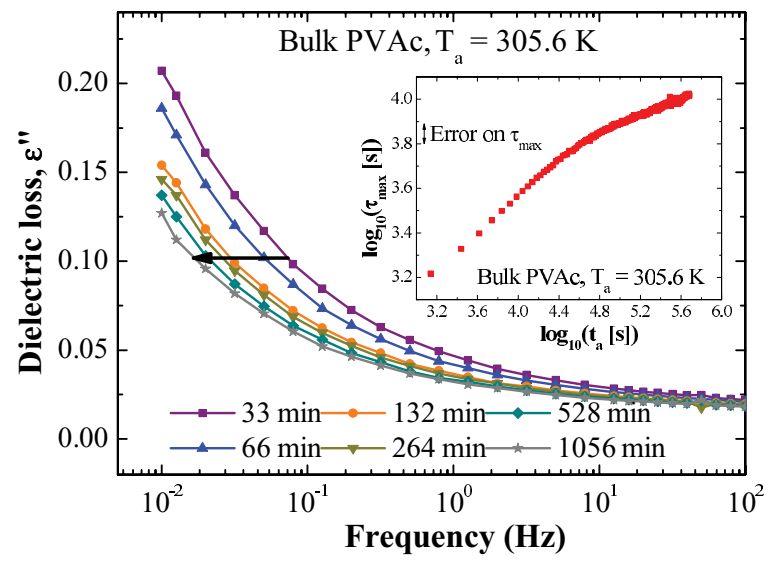

FIG. 5. (Color online) Frequency dependence of the loss part of the permittivity as a function of the aging time at $305.6 \mathrm{~K}$ for pure PVAc. Inset: Aging time evolution of the segmental relaxation time obtained by fitting the dielectric spectra through the HN function [Eq. (3)].

onset of out-of-equilibrium effects when cooling down, this result, similarly to the enthalpy recovery results, indicates that PVAc-SiO ${ }_{2}$ nanocomposites are able to maintain equilibrium more easily than the corresponding bulk PVAc.

\section{B. Segmental relaxation time during physical aging}

Figure 5 shows the frequency-dependent dielectric loss at different aging times at $305.6 \mathrm{~K}$ for bulk PVAc as an example. During the course of aging the dielectric loss evolves in a way that can be interpreted as a change in the dielectric strength, a change in the shape of the relaxation, and/or a shift in the mean relaxation time. Considering the equilibrium values of the dielectric strength and the HN shape parameters—obtained by extrapolating them with a linear function of temperature from above $T_{g}$-and those at the beginning of the aging process-corresponding to the values at $T_{g}$ - one can estimate a change in the dielectric strength and the $\mathrm{HN}$ shape parameters of the order of a few percent. Conversely, considering the temperature dependence of the equilibrium relaxation time according to the VFT law, it is possible to estimate a change in the relaxation time during the course of aging of between 0.5 and 1 order of magnitude, depending on the aging temperature. According to the previous arguments, one can safely conclude that the shift of the relaxation time is the main factor responsible for the evolution of the dielectric loss during aging. This conclusion is general since, as shown previously, PVAc and its nanocomposites with silica investigated here exhibit identical relaxation time distributions. Thus, fixing the dielectric strength and shape parameters in the HN equation

TABLE I. $T_{g}^{\text {on }}$ values, determined at the cooling rates of 20 and $0.2 \mathrm{~K} \mathrm{~min}^{-1}$, and time to reach equilibrium, $t_{\infty}$, at all investigated aging temperatures for bulk PVAc and its nanocomposites with $\mathrm{SiO}_{2}$.

\begin{tabular}{|c|c|c|c|c|c|}
\hline \multirow[b]{2}{*}{ Sample } & \multirow{2}{*}{$\begin{array}{c}T_{g}^{\mathrm{on}}\left(20 \mathrm{~K} \mathrm{~min}^{-1}\right) \\
(\mathrm{K})\end{array}$} & \multirow{2}{*}{$\begin{array}{c}T_{g}^{\mathrm{on}}\left(0.2 \mathrm{~K} \mathrm{~min}^{-1}\right) \\
(\mathrm{K})\end{array}$} & \multicolumn{3}{|c|}{$\log _{10}\left[t_{\infty}(\mathrm{s})\right]$} \\
\hline & & & $309.1 \mathrm{~K}$ & $308.1 \mathrm{~K}$ & $305.6 \mathrm{~K}$ \\
\hline PVAc & $319 \pm 1$ & $315 \pm 1$ & 4.65 & 5.15 & 6.15 \\
\hline V8 & $317 \pm 1$ & $313 \pm 1$ & 4.2 & 4.8 & 5.85 \\
\hline V25 & $315 \pm 1$ & $311 \pm 1$ & 3.9 & 4.5 & 5.4 \\
\hline
\end{tabular}



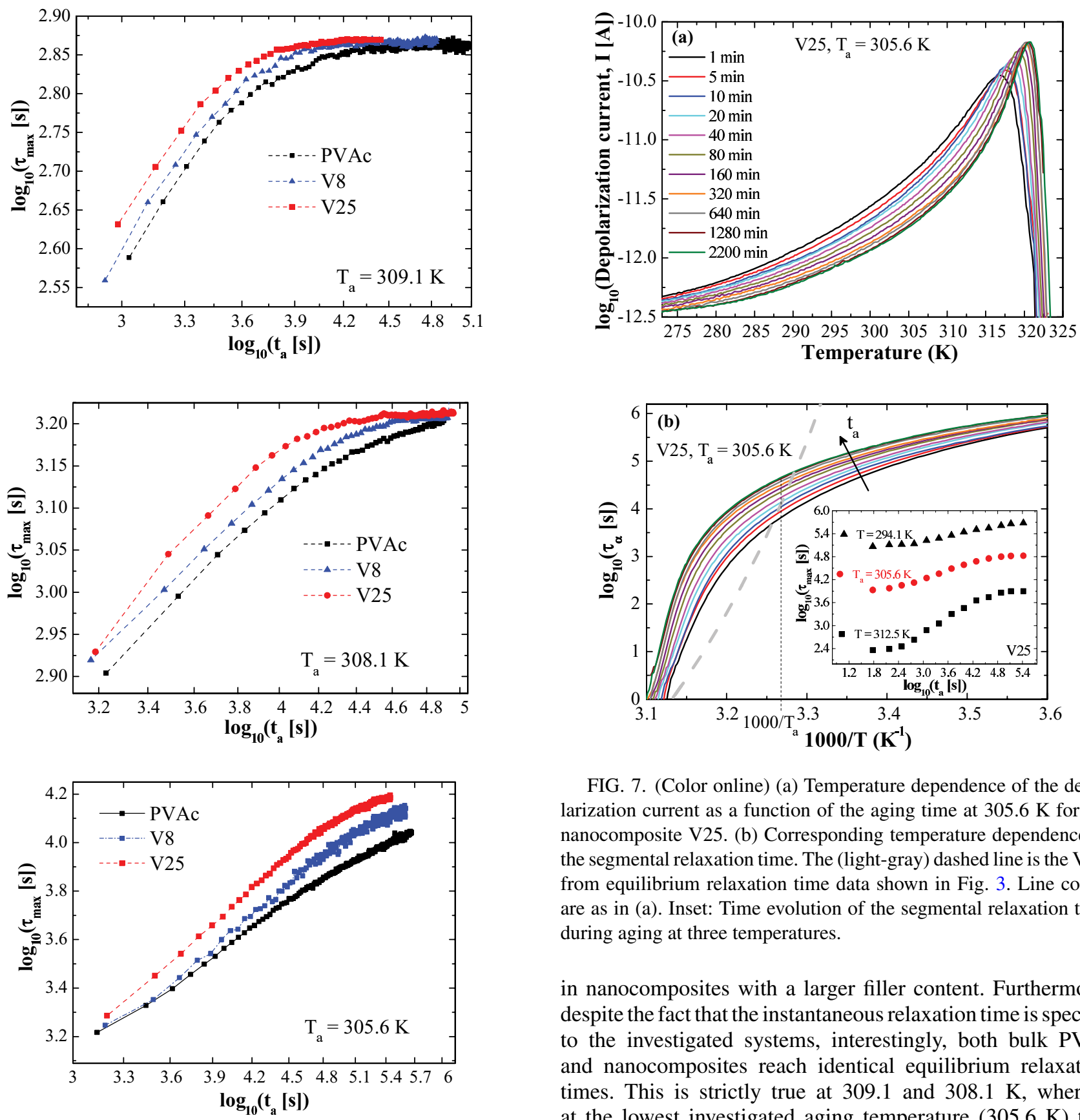

FIG. 6. (Color online) Aging-time evolution of the segmental relaxation time obtained from BDS at the indicated temperatures for all investigated systems.

[Eq. (3)] to those corresponding to the equilibrium, it is possible to extract the time evolution during physical aging of the segmental relaxation time. This is shown in the inset in Fig. 5 for bulk PVAc at $305.6 \mathrm{~K}$. Employing this procedure, the maximum error in the determination of the segmental relaxation time was evaluated to be about $10 \%$, as shown in the inset in Fig. 5.

A comprehensive view of the time evolution of the segmental relaxation time probed by BDS is provided in Fig. 6. Inspection of the figure reveals a faster evolution of the segmental relaxation time of the nanocomposites in comparison to pure PVAc, this being more pronounced

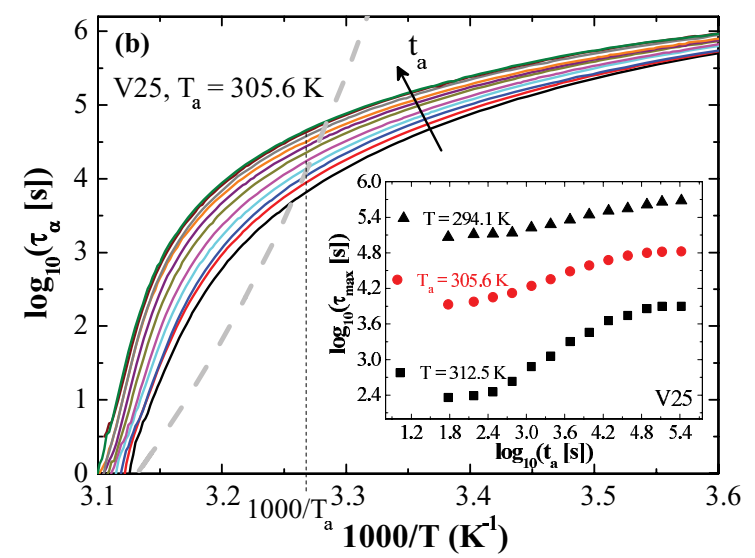

FIG. 7. (Color online) (a) Temperature dependence of the depolarization current as a function of the aging time at $305.6 \mathrm{~K}$ for the nanocomposite V25. (b) Corresponding temperature dependence of the segmental relaxation time. The (light-gray) dashed line is the VFT from equilibrium relaxation time data shown in Fig. 3. Line colors are as in (a). Inset: Time evolution of the segmental relaxation time during aging at three temperatures.

in nanocomposites with a larger filler content. Furthermore, despite the fact that the instantaneous relaxation time is specific to the investigated systems, interestingly, both bulk PVAc and nanocomposites reach identical equilibrium relaxation times. This is strictly true at 309.1 and $308.1 \mathrm{~K}$, whereas at the lowest investigated aging temperature $(305.6 \mathrm{~K})$ this conclusion cannot be reached unambiguously, since the aging time needed to reach equilibrium is too long in comparison to the aging time considered in the present work.

A different way to monitor the aging-time dependence of the segmental relaxation time is by means of TSDC. Figure 7(a) displays the temperature dependence of the depolarization current, measured at a heating rate of $10 \mathrm{~K} \mathrm{~min}^{-1}$, of the nanocomposite V25 after annealing at $305.6 \mathrm{~K}$ for the indicated aging time. From examination of the figure, it is straightforward to conclude that the maximum of the depolarization current shifts to higher temperatures with increasing aging time.

As previously discussed, the shape of the TSDC curves is intimately related to the temperature dependence of the segmental relaxation time (cf. Fig. 2). In Fig. 7(b) the temperature dependence of the segmental relaxation time 

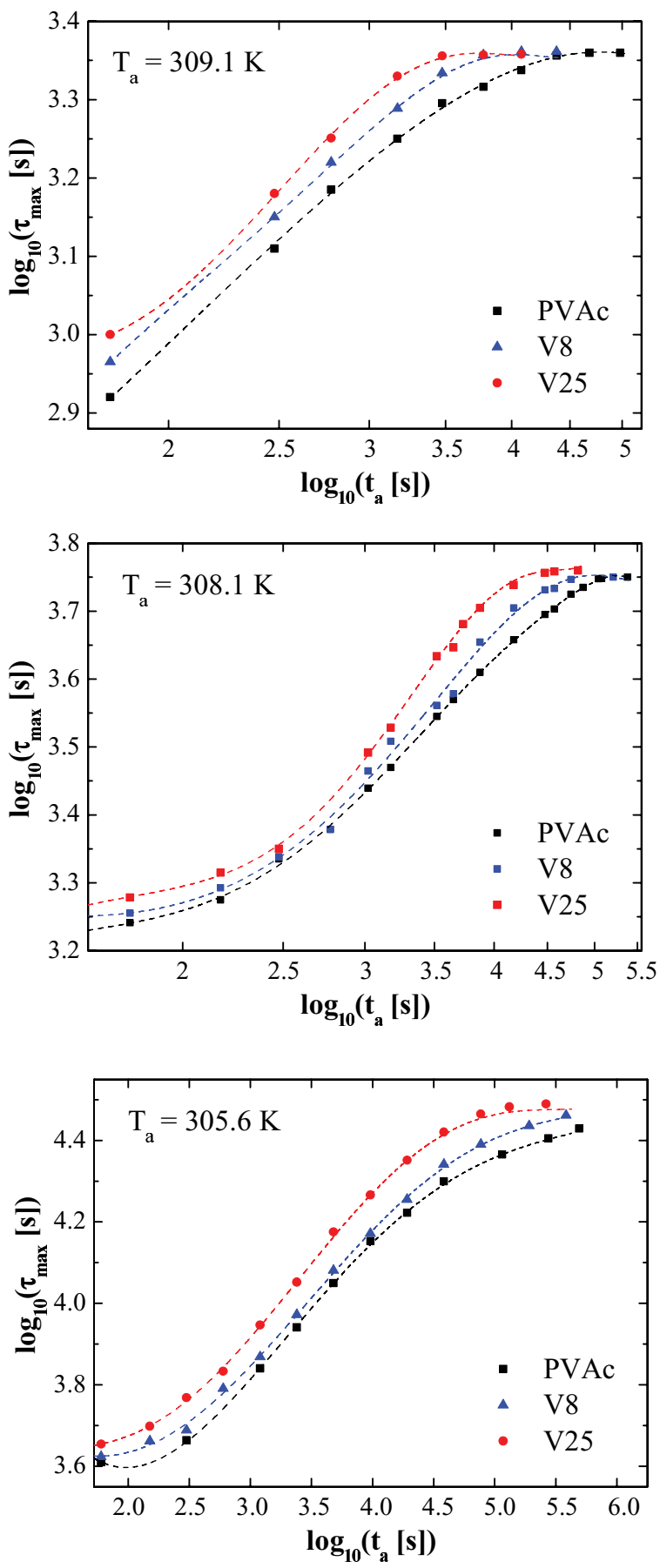

FIG. 8. (Color online) Aging-time evolution of the segmental relaxation time obtained from TSDC at the indicated temperatures for all investigated systems.

corresponding to the depolarization current, displayed in Fig. 7(a) and calculated through Eq. (2), is shown as a function of the annealing time at $305.6 \mathrm{~K}$ for the sample V25. As can be seen, the segmental relaxation time exhibits an increase with the aging time, reaching a plateau in the long-aging-time limit. This is shown in detail in the inset in Fig. 7, where the time evolution during physical aging of the segmental relaxation time is presented for the nanocomposite V25 at different temperatures.

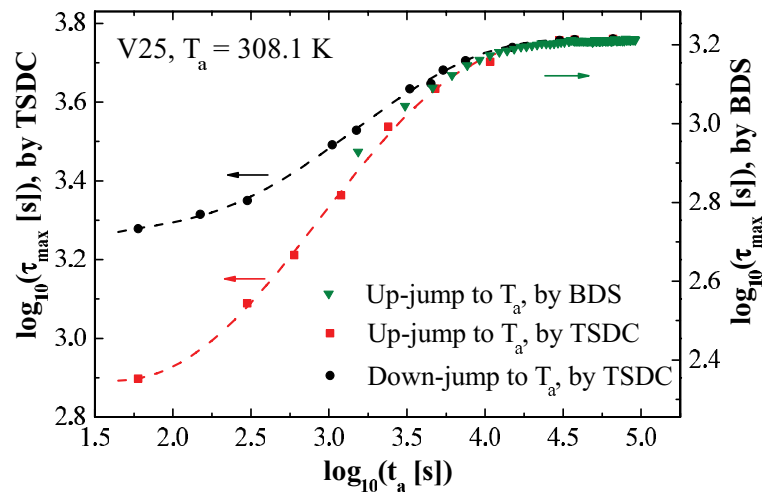

FIG. 9. (Color online) Comparison between the aging-time evolution of the segmental relaxation time obtained from TSDC following two different thermal histories for the sample V25 at 308.1 K. The corresponding segmental relaxation time obtained by BDS is also displayed.

The time evolution due to physical aging of the instantaneous segmental relaxation time at the aging temperature obtained from TSDC experiments is reported in Fig. 8. These results are analogous to those obtained by BDS and can be summarized as follows: (i) the segmental relaxation time at a given aging temperature increases more rapidly for nanocomposites than for bulk PVAc; and (ii) after long-term aging, all systems reach the same segmental relaxation times. It is noteworthy that the absolute value of the segmental relaxation time obtained by TSDC is not an equilibrium relaxation time since it is monitored during a continuous heat scan. Particularly, after reaching equilibrium at the annealing temperature the sample is affected by the nonisothermal aging occurring during the subsequent cooling-heating cycle (cf. Fig. 2). Thus the limiting value of the segmental relaxation time is expected to be larger than that obtained by BDS. This is actually found comparing Fig. 8 with Fig. 6, where the TSDC segmental relaxation time at the annealing temperature is larger than that found by BDS (less than 1 order of magnitude). Another significant difference between TSDC and BDS experiments is the fact that the thermal history of samples whose aging is monitored by BDS is not the same as that employed in TSDC. In particular, as detailed in Sec. II, in the case of BDS, samples are first quenched at temperatures considerably lower than $T_{g}$ and subsequently heated up at the selected aging temperature, whereas samples are directly brought to a given aging temperature in the case of TSDC. This implies a more marked nonisothermal aging during TSDC measurements in comparison to BDS ones, thereby explaining the smoother short annealing time dependences in the case of TSDC in comparison to BDS. This interpretation is corroborated by the results of the aging-time evolution of the segmental relaxation time from TSDC following the temperature protocol used for BDS. As can be seen in Fig. 9, where this time evolution is displayed for V25 at 308.1 $\mathrm{K}$, the aging-time dependence of the segmental relaxation time by TSDC mirrors that of the same sample obtained by BDS.

The limiting values of the segmental relaxation times obtained from BDS are reported in Fig. 3(b) together with those obtained at higher temperatures. As can be observed, the VFT 
fit of the latter accurately catches the temperature dependence of the relaxation time also at lower temperatures, in particular, those relevant for our aging experiments. The VFT line is also included in Fig. 7(b). From examination of this figure, it is clear that the limiting values of the segmental relaxation time obtained by TSDC at a given annealing temperature are larger than those corresponding to equilibrium.

\section{DISCUSSION}

In the previous sections, both thermodynamic and dynamic aspects of the physical aging of $\mathrm{PVAc}-\mathrm{SiO}_{2}$ nanocomposites have been presented. The former indicate the intimate link between the area-to-volume ratio of the nanocomposites and the acceleration of physical aging in these systems in comparison to the bulk. This result, together with those found in other polymer nanocomposites $[12-15,40]$ and thin films [7,41-45] (the latter exhibiting, in analogy to nanocomposites, large interfaces), suggests a universal link between the areato-volume ratio and the rate of physical aging, at least in systems presenting weak interactions at the interface. ${ }^{1}$ The most obvious dilemma arising from these results is whether the relation between the amount of interface and the acceleration of physical aging is due to enhanced segmental dynamics or other factors beyond the molecular mobility. The traditional view of physical aging is that this phenomenon is related one-to-one to the intrinsic molecular mobility of the glass $[5,6]$. However, our recent studies suggested the breakdown of the validity of this relation in nanostructured glasses [12-14,40]. In those studies it was shown that physical aging in nanocomposites and thin films was enhanced in comparison to the bulk, despite the invariance of the segmental dynamics at equilibrium above $T_{g}$. An important concern of these previous studies was that the temperature range relevant for physical aging is significantly lower than that explored to determine the polymer segmental dynamics. Thus, the possibility of a dramatic change in the segmental dynamics at temperatures lower than those investigated, in particular, approaching those employed for physical aging studies, could be put forward. In the present study, by monitoring the agingtime dependence of the segmental relaxation time through BDS and TSDC, we were able to provide unambiguous arguments indicating that accelerated aging does not result from enhanced segmental dynamics, but rather depends on other factors. This statement originates from the following experimental results: (i) $\mathrm{PVAc}_{-} \mathrm{SiO}_{2}$ nanocomposites evolve toward equilibrium more rapidly than bulk PVAc, despite the fact that the instantaneous segmental relaxation time is larger in nanocomposites during the entire physical aging process; and (ii) the equilibrium segmental relaxation time achieved after prolonged aging is identical in all investigated systems. Thus we have demonstrated that the equilibration time cannot be exclusively related to the segmental dynamics but also depends on a factor related to the amount of interface in the nanostructured glass. Therefore the relation put forward

\footnotetext{
${ }^{1}$ In systems displaying strong interactions at the interface the observed alteration of the physical aging behavior may not be present as shown, for instance, in Refs. [61-64].
}

in Sec. I, $\tau_{\mathrm{PA}}=\tau_{\alpha} f_{A / V}$, is generally proved by the present results. Having clarified this important point, the open question regards the physics behind the function $f_{A / V}$.

Possible arguments invoking a change in the local chain packing as well as a longer range structural correlation may, in principle, be invoked to describe $f_{A / V}$. It is in fact well known that the structure of PVAc may be deeply altered in heterogeneous systems [46]. However, in the case of the present study the typical interparticle distances are of the order of several hundred nanometers and significant alterations of PVAc structure are likely negligible. Furthermore, even if present, modifications of the structure are not significant enough to induce a change in the polymer segmental dynamics, as shown by the results of the present study. A suitable framework to describe the physics behind $f_{A / V}$ relies on the employment of a diffusion mechanism of equilibration of nanostructured glasses, originally introduced to describe physical aging in bulk glasses [47] and later developed to describe the out-of-equilibrium dynamics in thin films [17,44,48,49]. Details about the development of this approach can be found elsewhere, where the model based on diffusion arguments is applied to both physical aging and $T_{g}$ depression data on polymer nanocomposites [13,50] and thin films [17]. Here we simply emphasize that, within this model, the densification of the nanostructured systems is a consequence of a hole diffusion mechanism, intimately related to the molecular mobility, and their disappearance at the interface. Thus, it becomes immediately clear that glasses exhibiting identical molecular mobilities but different amounts of interface display different rates of physical aging. We also remark that such a mechanism appears to be relevant in nanostructured systems displaying a volume-to-area ratio of the order of several microns or lower, since no size effects are observed in the physical aging of glasses with a typical size larger than several microns $[7,13,14,44]$. In nanocomposites the diffusion mechanism of equilibration would be assisted by cavitation phenomena at the polymer-filler interface [51]. Such phenomena, if present, would create a larger "free" surface for efficient removal of volume holes.

Another result that is important to emphasize is that the segmental relaxation time of PVAc and its nanocomposites reaches the same value at equilibrium. This suggests that all systems also reach the same limiting enthalpy value and implies that the lower recovered enthalpy in systems with a large area-to-volume ratio $[13,17,40]$ is mainly due to the nonisothermal aging occurring in the cooling step from above $T_{g}$ to a given aging temperature. This interpretation is supported by the increase in the amount of recovered enthalpy with the applied cooling rate in polystyrene-gold nanocomposites [17]. Further proof of the prominent role of nonisothermal aging with increasing area-to-volume ratio is provided by the permeability measurements by Paul and coworkers indicating a reduced permeability (i.e., higher density) at the beginning of the aging process for thin films [52] with a decrease in thickness. Furthermore, our TSDC experiments performed with two different temperature stabilization procedures, highlighting and minimizing the role of nonisothermal aging (cf. Fig. 9), respectively, indicate that in the former case a considerably smaller segmental relaxation time recovery is recorded. 
Finally, an important observation regards the temperature dependence of the segmental relaxation time, which can be well described by a single VFT equation over the entire investigated range [see Fig. 3(b)], i.e., for relaxation times as large as $\tau_{\alpha}=10^{5} \mathrm{~s}$. This result agrees with a previous study on the segmental dynamics of PVAc at relatively low temperatures [53], whereas it contrasts with the deviation from the VFT law found in a number of glass forming systems including polymers [44,54-59] and, very recently, also PVAc [60] at temperatures somewhat lower than those of the present study and Ref. [53].

\section{CONCLUSIONS}

We have performed a detailed investigation of the physical aging of PVAc and its nanocomposites with silica. Enthalpy recovery, in agreement with previous studies, was found to be faster in nanocomposites. A similar result was obtained for the time evolution of the segmental relaxation time. Furthermore, all investigated systems reached the same equilibrium segmental relaxation time. Thus the most important outcome of our investigation is that nanocomposites age more rapidly despite their longer instantaneous segmental relaxation times in comparison to the bulk. This result can be understood only by assuming that, apart from the segmental mobility, the rate of physical aging is also determined by other factors such as the amount of available interface in nanocomposites. This assumption is corroborated by the observation of accelerated aging in other nanocomposites and thin polymer films, i.e., systems also displaying a high area-to-volume ratio. We propose a diffusion-based mechanism as a possible framework to account for the acceleration of aging and the observed time evolution of the segmental relaxation time.

\section{ACKNOWLEDGMENTS}

The authors acknowledge the University of the Basque Country and Basque Country Government (Ref. No. IT436-07, Departamento de Educación, Universidades e Investigación; and the Spanish Minister of Education (Grant No. MAT 2007-63681) for their financial support. V.M.B. acknowledges the CSIC for the JAE-Doc contract, cofinanced by the European Social Fun. Energy Strategy Associates Inc. is acknowledged for kindly providing the silica nanoparticles.
[1] D. R. Paul and L. M. Robeson, Polymer 49, 3187 (2008).

[2] F. Hussain, M. Hojjati, M. Okamoto, and R. E. Gorga, J. Compos. Mater. 40, 1511 (2006).

[3] A. Kutvonen, G. Rossi, and T. Ala-Nissila, Phys. Rev. E 85, 041803 (2012).

[4] I. M. Hodge, Science 267, 1945 (1995).

[5] A. J. Kovacs, Fortschr Hochpolym.-Forsch 3, 394 (1963).

[6] L. C. E. Struik, Physical Aging in Amorphous Glassy Polymers and Other Materials, 1st ed. (Elsevier Science, Amsterdam, 1978).

[7] Y. Huang and D. R. Paul, Polymer 45, 8377 (2004).

[8] I. M. Hodge, J. Non-Cryst. Solids 169, 211 (1994).

[9] J. M. Hutchinson, Prog. Polym. Sci. 20, 703 (1995).

[10] R. D. Priestley, P. Rittigstein, L. J. Broadbelt, K. Fukao, and J. M. Torkelson, J. Phys.: Condens. Matter. 19, 2996 (2007).

[11] R. D. Priestley, Soft Matter 5, 919 (2009).

[12] V. M. Boucher, D. Cangialosi, A. Alegría, J. Colmenero, J. Gonzalez-Irun, and L. M. Liz-Marzan, Soft Matter 6, 3306 (2010).

[13] V. M. Boucher, D. Cangialosi, A. Alegría, J. Colmenero, I. Pastoriza-Santos, and L. M. Liz-Marzan, Soft Matter 7, 3607 (2011).

[14] D. Cangialosi, V. M. Boucher, A. Alegria, and J. Colmenero, Polymer 53, 1362 (2012).

[15] F. Barroso-Bujans, V. M. Boucher, J. A. Pomposo, L. Buruaga, A. Alegría, and J. Colmenero, Chem. Commun. 48, 2618 (2012).

[16] V. M. Boucher, D. Cangialosi, A. Alegría, and J. Colmenero, AIP Conf. Proc. 1255, 172 (2010).

[17] V. M. Boucher, D. Cangialosi, A. Alegría, and J. Colmenero, Macromolecules 45, 5296 (2012).

[18] G. Williams and D. Watts, Trans. Faraday Soc. 66, 80 (1970).

[19] L. Goitiandia and A. Alegria, J. Non-Cryst. Solids 287, 237 (2001).
[20] M. Tyagi, A. Alegria, and J. Colmenero, J. Chem. Phys. 122, 244909 (2005).

[21] A. Alegria, L. Goitiandia, and J. Colmenero, Polymer 37, 2915 (1996).

[22] F. Alvarez, A. Alegria, and J. Colmenero, Phys. Rev. B 44, 7306 (1991).

[23] E. Krygier, G. Lin, J. Mendes, G. Mukandela, D. Azar, A. Jones, J. Pathak, R. Colby, S. Kumar, G. Floudas, R. Krishnamoorti, and R. Faust, Macromolecules 38, 7721 (2005).

[24] G. Johari, J. Chem. Phys. 77, 4619 (1982).

[25] E. Schlosser and A. Schonhals, Polymer 32, 2135 (1991).

[26] A. Alegria, E. Guerrica-Echevarria, L. Goitiandia, I. Telleria, and J. Colmenero, Macromolecules 28, 1516 (1995).

[27] A. Alegria, L. Goitiandia, I. Telleria, and J. Colmenero, Macromolecules 30, 3881 (1997).

[28] L. Goitiandia and A. Alegria, J. Chem. Phys. 121, 1636 (2004).

[29] P. Lunkenheimer, R. Wehn, U. Schneider, and A. Loidl, Phys. Rev. Lett. 95, 055702 (2005).

[30] K. Fukao and D. Tahara, Phys. Rev. E 80, 051802 (2009).

[31] R. Casalini and C. M. Roland, Phys. Rev. Lett. 102, 035701 (2009).

[32] T. Hecksher, N. B. Olsen, K. Niss, and J. C. Dyre, J. Chem. Phys. 133, 174514 (2010).

[33] Z. Wojnarowska, C. M. Roland, K. Kolodziejczyk, A. SwietyPospiech, K. Grzybowska, and M. Paluch, J. Phys. Chem. Lett. 3, 1238 (2012).

[34] S. Havriliak and S. Negami, Polymer 8, 161 (1967).

[35] F. Alvarez, A. Alegria, and J. Colmenero, Phys. Rev. B 47, 125 (1993).

[36] R. B. Bogoslovov, C. M. Roland, A. R. Ellis, A. M. Randall, and C. G. Robertson, Macromolecules 41, 1289 (2008).

[37] G. J. Schneider, K. Nusser, L. Willner, P. Falus, and D. Richter, Macromolecules 44, 5857 (2011). 
[38] G. Schwartz, E. Tellechea, J. Colmenero, and A. Alegria, J. Non-Cryst. Solids 351, 2616 (2005).

[39] S. Amanuel, A. N. Gaudette, and S. S. Sternstein, J. Polym. Sci. Polym. Phys. Ed. 46, 2733 (2008).

[40] V. M. Boucher, D. Cangialosi, A. Alegría, and J. Colmenero, Macromolecules 43, 7594 (2010).

[41] M. S. McCaig and D. R. Paul, Polymer 41, 629 (2000).

[42] K. D. Dorkenoo and P. H. Pfromm, Macromolecules 33, 3747 (2000).

[43] C. Zhou, T. S. Chung, R. Wang, and S. H. Goh, J. Appl. Polym. Sci. 92, 1758 (2004).

[44] D. Cangialosi, M. Wübbenhorst, J. Groenewold, E. Mendes, H. Schut, A. van Veen, and S. J. Picken, Phys. Rev. B 70, 224213 (2004).

[45] B. W. Rowe, S. J. Pas, A. J. Hill, R. Suzuki, B. D. Freeman, and D. R. Paul, Polymer 50, 6149 (2009).

[46] G. Zardalidis and G. Floudas, Macromolecules 45, 6272 (2012); [http://pubs.acs.org/doi/pdf/10.1021/ma301086a].

[47] J. G. Curro, R. R. Lagasse, and R. Simha, Macromolecules 15 , 1621 (1982).

[48] M. S. McCaig, D. R. Paul, and J. W. Barlow, Polymer 41, 639 (2000).

[49] A. W. Thornton and A. J. Hill, Ind. Eng. Chem. Res. 49, 12119 (2010).

[50] D. Cangialosi, V. M. Boucher, A. Alegría, and J. Colmenero, J. Chem. Phys. 135, 014901 (2011).
[51] B. Ash, D. Rogers, C. Wiegand, L. Schadler, R. Siegel, B. Benicewicz, and T. Apple, Polym. Compos. 23, 1014 (2002).

[52] B. W. Rowe, B. D. Freeman, and D. R. Paul, Polymer 51, 3784 (2010).

[53] R. Richert, Physica A 287, 26 (2000).

[54] P. O'Connell and G. McKenna, J. Chem. Phys. 110, 11054 (1999).

[55] S. L. Simon, J. W. Sobieski, and D. J. Plazek, Polymer 42, 2555 (2001).

[56] K. Chen and S. Vyazovkin, J. Phys. Chem. B 113, 4631 (2009).

[57] T. Hecksher, A. I. Nielsen, N. B. Olsen, and J. C. Dyre, Nat. Phys. 4, 737 (2008).

[58] J. C. Mauro, D. C. Allan, and M. Potuzak, Phys. Rev. B 80, 094204 (2009).

[59] V. M. Boucher, D. Cangialosi, A. Alegria, and J. Colmenero, Macromolecules 44, 8333 (2011).

[60] J. Zhao and G. B. McKenna, J. Chem. Phys. 136, 154901 (2012).

[61] A. Y. H. Liu and J. Rottler, J. Polym. Sci., Part B: Polym. Phys. 47, 1789 (2009).

[62] Y. Guo, C. Zhang, C. Lai, R. D. Priestley, M. D'Acunzi, and G. Fytas, ACS Nano 5, 5365 (2011).

[63] T. M. Murphy, D. S. Langhe, M. Ponting, E. Baer, B. D. Freeman, and D. R. Paul, Polymer 52, 6117 (2011).

[64] D. S. Langhe, T. M. Murphy, A. Shaver, C. LaPorte, B. D. Freeman, D. R. Paul, and E. Baer, Polymer 53, 1925 (2012). 